\title{
ESTUDO DO ESCOAMENTO DE GÁS NATURAL E FORMAÇÃO DE ENXOFRE ELEMENTAR EM UMA VÁLVULA REDUTORA DE PRESSÃO
}

\author{
E.R. BRAGA ${ }^{1}$, C. MORAES ${ }^{1}$ e R. A. MEDRONHO ${ }^{1}$ \\ ${ }^{1}$ Universidade Federal do Rio de Janeiro, Departamento de Engenharia Química \\ E-mail para contato: erbraga@eq.ufrj.br
}

\begin{abstract}
RESUMO - Válvulas redutoras de pressão de gás são amplamente utilizadas nos sistemas de produção e transporte de gás natural, sendo este gás uma mistura multicomponente de hidrocarbonetos (composto principalmente de metano e etano), dióxido de carbono, nitrogênio e traços de enxofre. $\mathrm{O}$ gás apresenta geralmente um comportamento de fases complexo. A região do diagrama de fases onde ocorre condensação de líquido, por redução da pressão ou aumento da temperatura, oposto ao comportamento normal é chamada de região de condensação retrógrada, e é identificada pelo aparecimento de gotículas suspensas na corrente de gás. Neste trabalho foram realizadas simulações utilizando fluidodinâmica computacional a fim de caracterizar o escoamento de gás natural em uma válvula redutora de pressão. Foi estudada também a deposição de enxofre através dos conceitos da teoria clássica da nucleação.
\end{abstract}

\section{INTRODUÇÃO}

O gás natural, por se tratar de uma mistura multicomponente, apresenta geralmente um comportamento de fases complexo. A região do diagrama de fases onde a condensação do líquido ocorre, por redução da pressão ou aumento da temperatura, oposto ao comportamento normal, é chamada de região de condensação retrógrada, identificada pelo aparecimento de gotículas suspensas na corrente de gás. O enxofre, inicialmente presente na fase gasosa, é dissolvido nestas gotículas formadas (fase líquida). Desta forma, ao ocorrer a evaporação das gotículas, o enxofre elementar dissolvido torna-se supersaturado e coagula formando partículas sólidas que servem de sementes para o início do processo de nucleação heterogênea.

O fenômeno da deposição de enxofre elementar em dutos de transporte de gás natural pode ser estudado através dos conceitos da nucleação pode ter sua influência estudada com a aplicação dos conceitos da teoria clássica da nucleação. Para o transporte de gás natural, a condensação retrógrada possui maiores chances de ocorrer quando o gás contém hidrocarbonetos pesados em sua composição. Estes componentes são mais favoráveis a estarem presentes na fase líquida do que os hidrocarbonetos leves. Caso ocorra uma redução de temperatura na corrente de gás é provável que gotículas se formem e que contenham uma alta fração de hidrocarbonetos pesados. Com isso, a partir dos diagramas de fases, obtidos para diferentes composições de gás natural e com distintas frações de hidrocarbonetos pesados, a influência deste parâmetro poderá ser avaliada. 


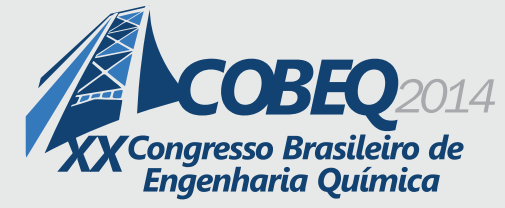

A nucleação é um processo durante o qual pequenos núcleos crescem e se dispersam, de modo a atingirem um determinado tamanho (núcleo crítico) que possibilita o crescimento contínuo do cristal. A nucleação é um fenômeno microscópico que envolve de dezenas a milhares de moléculas, o que torna difícil sua observação em dutos de transporte de gás.

A região do diagrama de fases onde ocorre condensação de líquido, por redução da pressão ou aumento da temperatura, oposto ao comportamento normal, é chamada de região de condensação retrógrada e é identificada pelo aparecimento de gotículas suspensas na corrente de gás.

Em uma válvula redutora de pressão é necessário caracterizar bem o escoamento do gás natural a fim de entender melhor o fenômeno da nucleação. Para este fim foi utilizada a técnica de Fluidodinâmica Computacional.

\section{REVISÃO BIBLIOGRÁFICA}

Zhu et al. (2011) afirmam que no momento em que a concentração inicial de enxofre na fase vapor atinge o valor da sua solubilidade no gás natural, a precipitação de enxofre elementar não ocorre imediatamente. Um limite desta barreira, controlado pela energia livre de Gibbs, necessita ser ultrapassado, com base na teoria clássica da nucleação. Segundo Turk (2000), a taxa de nucleação pode ser obtida a partir da combinação das Equações (1) a (5):

$J=K e^{\left(-\frac{\Delta G}{k_{B} T}\right)}$

$K=k_{0} \alpha_{c} v_{S} N^{2}\left(\frac{2 \sigma}{k_{B} T}\right)^{\frac{1}{2}}$

$v_{S}=\left\{M\left[3-2\left(\frac{T}{T_{b}}\right)\right]^{0,31} N_{A}\right\}^{-1}$

$N=\rho_{M} y_{E} N_{A}$

$\sigma=P_{c}^{2 / 3} T_{c}{ }^{1 / 3}\left[0,1207\left(1+\frac{T_{b r} \ln \left(P_{c}\right)}{1-T_{b r}}\right)-0,281\right]\left(1-T_{r}\right)^{1,222}$

A partir dessas equações, pode-se calcular a massa de enxofre que pode ser depositada através do processo de nucleação/dessublimação, em um determinado ponto, a partir do valor da taxa de formação de núcleos.

Em condições de redução de pressão, como nos casos das válvulas redutoras de pressão, temperaturas muito baixas podem ser alcançadas e as taxas de nucleação podem atingir valores muito maiores. Segundo Pack (2005), nucleação em determinadas restrições ao escoamento podem atingir valores da ordem de $1013 \mathrm{~cm}^{-3} \mathrm{~s}^{-1}$, o que justifica serem os locais à jusante destas válvulas os que apresentam a maior quantidade de enxofre elementar depositado. 


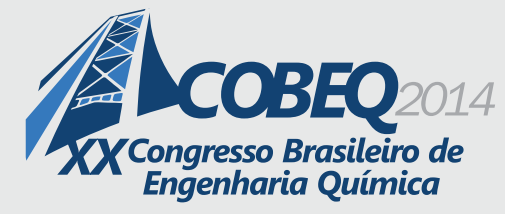

19 a 22 de outubro de 2014

Florianópolis/SC

\section{MATERIAIS E MÉTODOS}

A geometria em $C A D$ da válvula para a simulação foi construída utilizando o programa Design Modeler do pacote computacional Ansys Versão 13.0. Na construção desta geometria, foram rigorosamente obedecidas as dimensões da válvula redutora de pressão instalada em uma planta piloto da Universidade Federal de Itajubá. Esta válvula possui uma geometria bem complexa devido a existirem muitas restrições ao escoamento. A fim de fornecer uma melhor compreensão da geometria, são mostradas fotos da válvula na Figura 1.

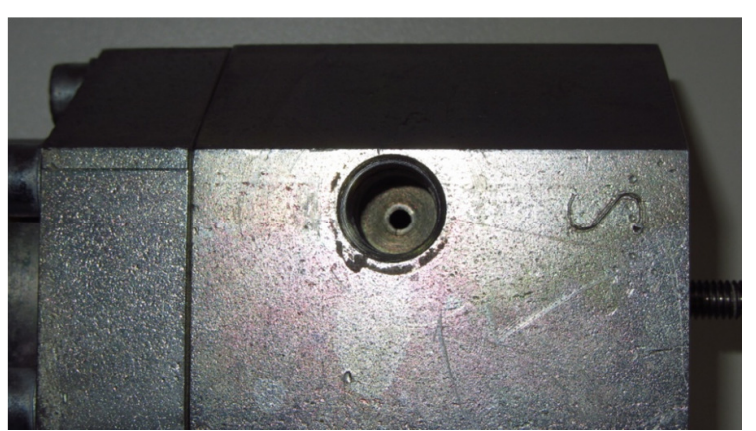

(a)

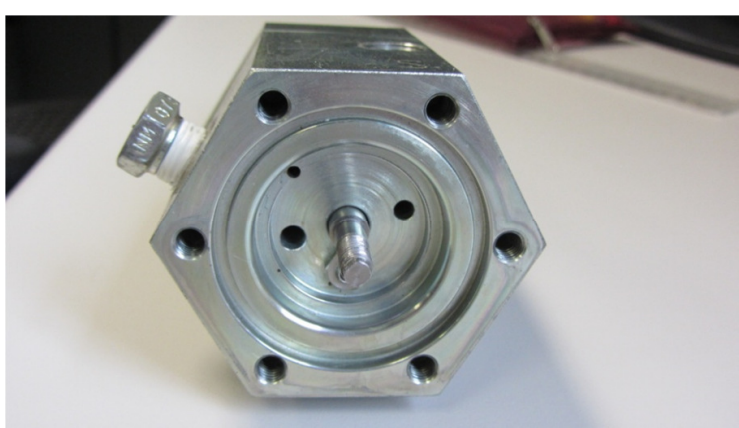

(b)

Figura 1 - Fotos da válvula

A Figura 1a mostra a parte externa da válvula com ênfase à região de entrada do fluido na válvula, onde se pode observar uma grande redução no diâmetro da entrada. A Figura 1b mostra a válvula aberta. Nela, pode-se observar a região de expansão do fluido. Há ainda um parafuso no meio da válvula que restringe o escoamento.

A Figura 2 mostra duas vistas da geometria construída no Design Modeller. A primeira é uma vista superior e a segunda uma vista lateral.

aNSSYS
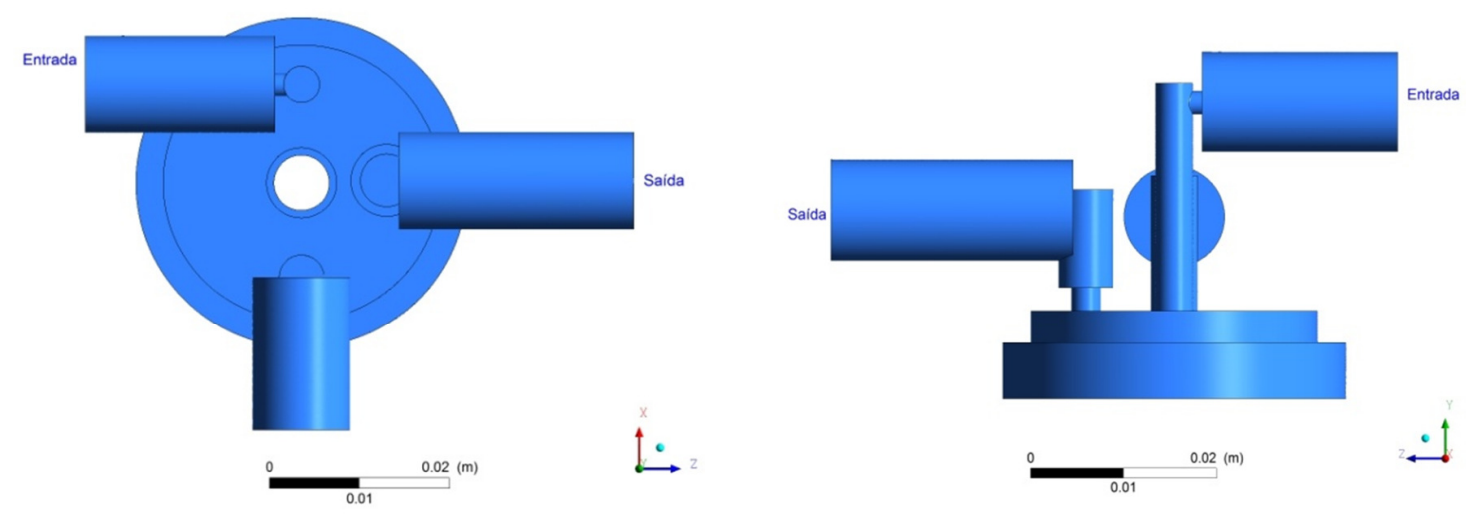

Figura 2 - Geometria em três vistas diferentes 
Deve-se ressaltar que o equipamento possui duas saídas, porém, normalmente opera com apenas uma saída, criando assim uma zona de morta de gás na válvula.

A construção da malha foi utilizando o programa Ansys Meshing Versão 13.0. Devido à complexidade da geometria, todas as malhas foram construídas com elementos tetraédricos com camadas de prismas (inflation) próximas à parede. Para as etapas de definição da simulação, solução das equações de conservação definidas do problema e análise dos resultados foram utilizados respectivamente os programas $C F X$-Pre, $C F X$-Solver e CFD-Post, ambos na versão 13.0.

Para o teste de independência da malha, foram construídas 8 malhas. A Tabela 1 mostra a quantidade de nós em cada malha.

Tabela 1 - Número de nós das diferentes malhas empregadas no teste de malha.

\begin{tabular}{cc}
\hline \hline Malha & Nós \\
\hline 1 & 58.224 \\
2 & 115.822 \\
3 & 347.924 \\
4 & 692.484 \\
5 & 1.334 .359 \\
6 & 2.144 .590 \\
7 & 2.392 .658 \\
8 & 2.506 .663 \\
\hline
\end{tabular}

Todas as simulações foram realizadas para regime estacionário, utilizando o modelo de turbulência $\kappa-\varepsilon$, com esquema de discretização high resolution. Para o teste de malha, a fim de facilitar a convergência, o fluído utilizado foi apenas metano e foi considerado gás ideal.

Como condições de contorno, foram atribuídas pressões de entrada e saída de 7,84x106 Pa e $3,72 \times 106 \mathrm{~Pa}$, respectivamente. Como critério de convergência, as simulações foram consideradas terem atingido a convergência quando os resíduos das equações de velocidade, pressão e turbulência atingiam valores menores que 0,001 e o mass imbalance gerava valores menores que 0,1. Para comparar as malhas, foram traçadas seis linhas em algumas posições dentro da válvula (Figura 3) onde foram medidas as velocidades do gás. 


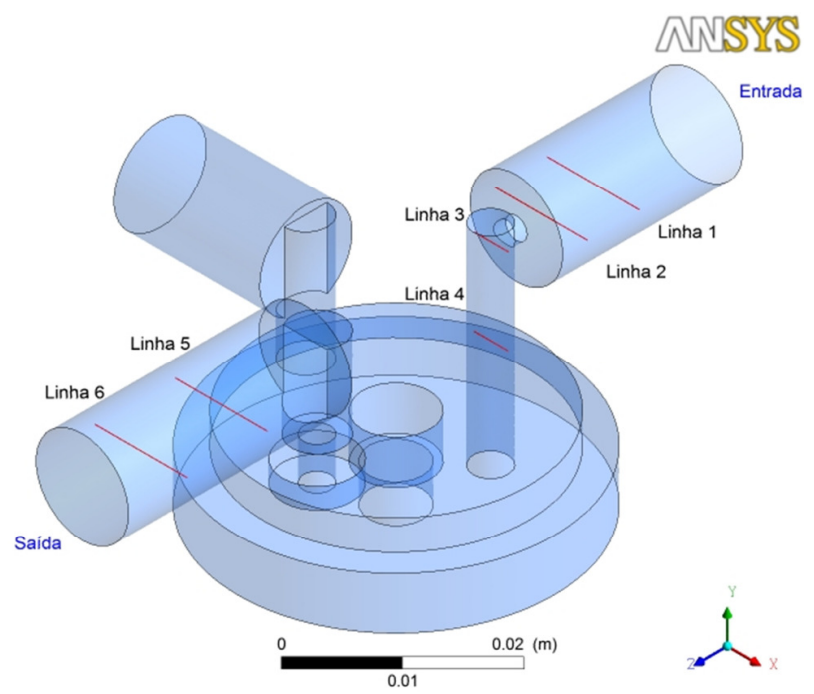

Figura 3 - Linhas para comparação de malhas

A figura a seguir mostra perfis de velocidades obtidos para a linha 2 em todas as malhas.

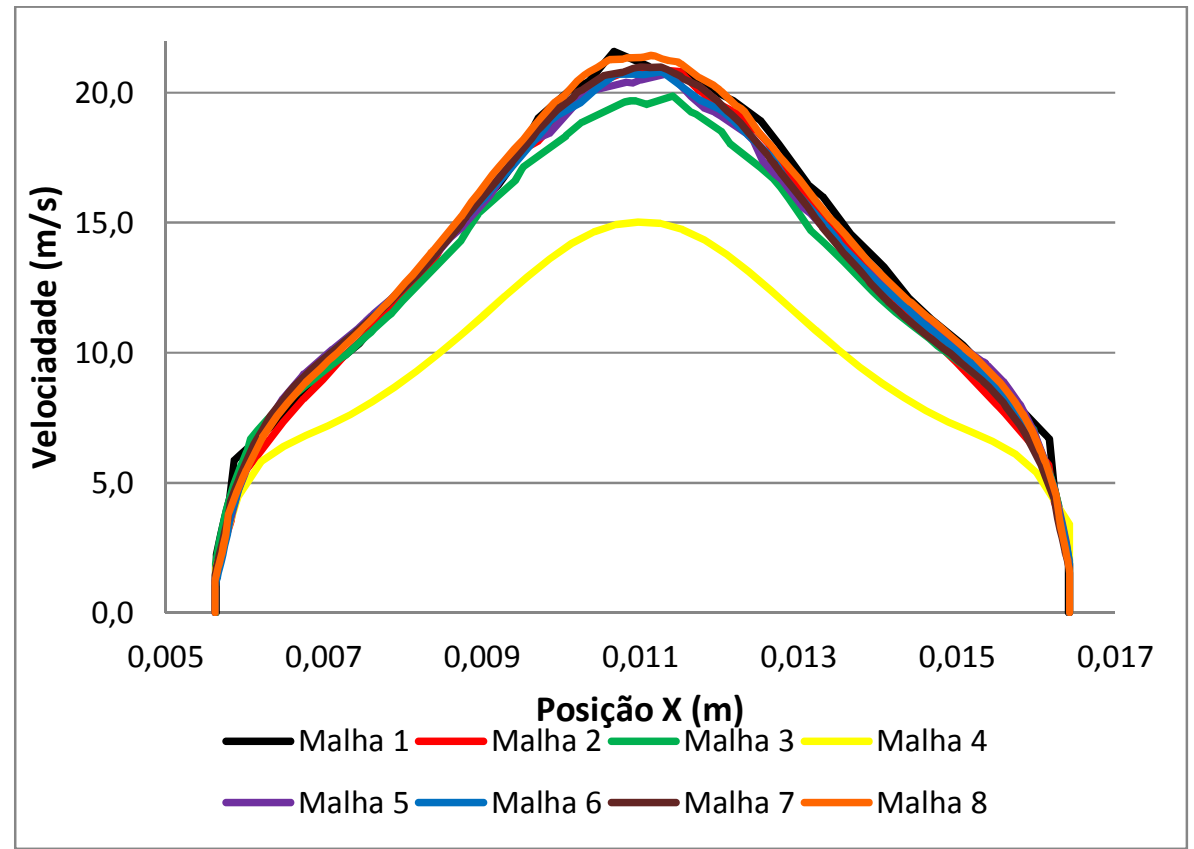

Figura 4 - Perfil de velocidade na linha 2

Pode-se observar que as malhas 7 e 8 possuem perfis bem semelhantes, por isso, a malha 7 foi adotada como melhor malha. Em seguida foi feita uma simulação com a malha 7 considerando o gás natural como gás ideal e uma mistura ideal de componentes cuja composição está apresentada na Tabela 2: 
Tabela 2. Composição do gás simulado.

\begin{tabular}{cc}
\hline \hline Componente & Fração molar (\%) \\
\hline Metano & 88,8 \\
Etano & 6,14 \\
Propano & 1,8 \\
Butano & 0,66 \\
Pentano & 0,19 \\
Hexano & 0,07 \\
CO2 & 1,54 \\
N2 & 0,8 \\
\hline \hline
\end{tabular}

As propriedades físicas, como viscosidade, densidade e peso molecular dos componentes foram obtidas através do banco de dados do próprio Ansys Cfx-Pre. As condições de contorno, modelagem matemática e critérios de convergência são os mesmos do teste de malha (pressões de entrada e saída de $7,84 \cdot 10^{6} \mathrm{~Pa}$ e $3,72 \cdot 10^{6} \mathrm{~Pa}$ respectivamente).

\section{RESULTADOS}

As figuras a seguir mostram resultados obtidos da simulação. A vazão mássica calculada foi de $0,0471 \mathrm{Kg} / \mathrm{s}$ e as velocidades médias do escoamento de gás natural na entrada e saída da válvula foram $10,32 \mathrm{~m} / \mathrm{s}$ e $23,04 \mathrm{~m} / \mathrm{s}$ respectivamente.

A Figura 5 exibe as linhas de corrente do escoamento do gás natural na válvula. Pode-se notar a existência de algumas zonas de recirculação do escoamento.

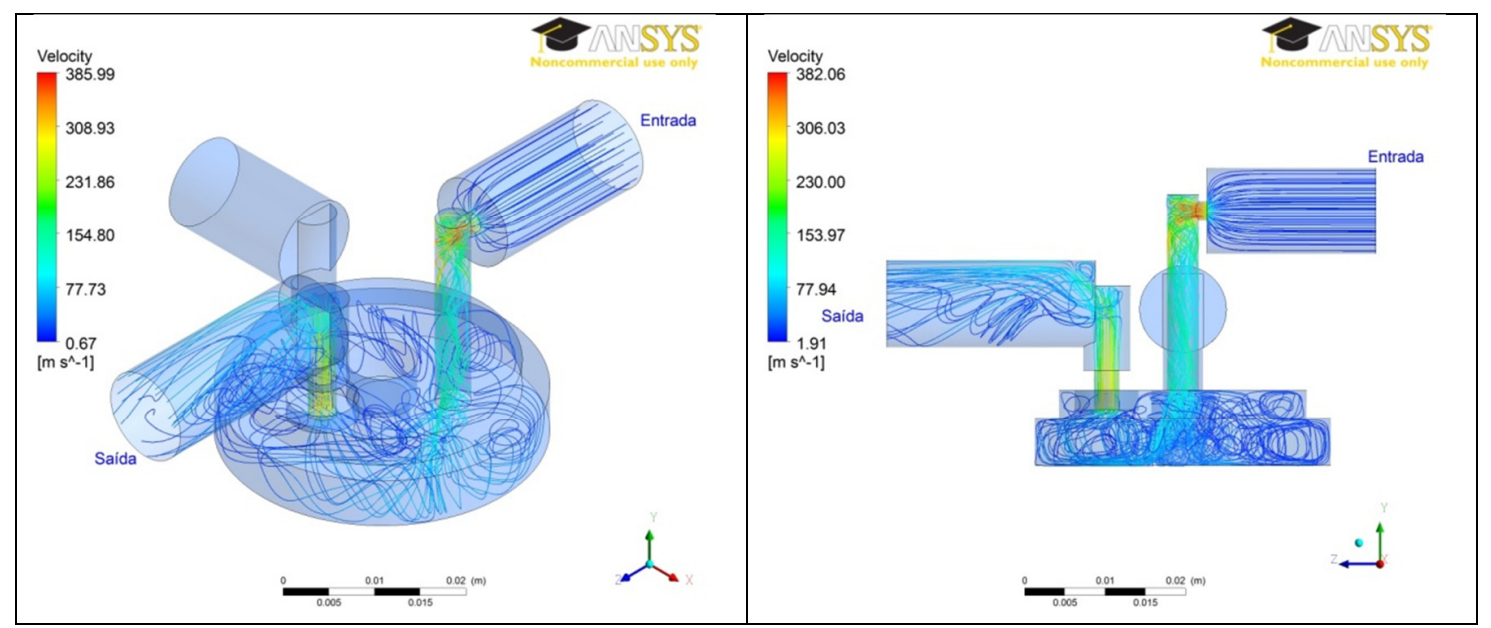

Figura 5 - Linhas de corrente 
A Figura 6 mostra o perfil de velocidade em dois planos distintos. O primeiro plano está localizado no centro da região de entrada e o segundo no centro da região de saída. Pode-se observar que, próximo à entrada, há uma brusca diminuição no diâmetro da válvula, o que faz com que o fluido atinja uma maior velocidade, obtendo um valor de número de Mach calculado próximo de 1. As duas regiões mais críticas com relação a altas velocidades estão localizadas no estrangulamento existente ao final da entrada e no tubo de subida do gás para a saída. Neste tubo, as velocidades chegam a atingir valores próximos de $370 \mathrm{~m} / \mathrm{s}$.

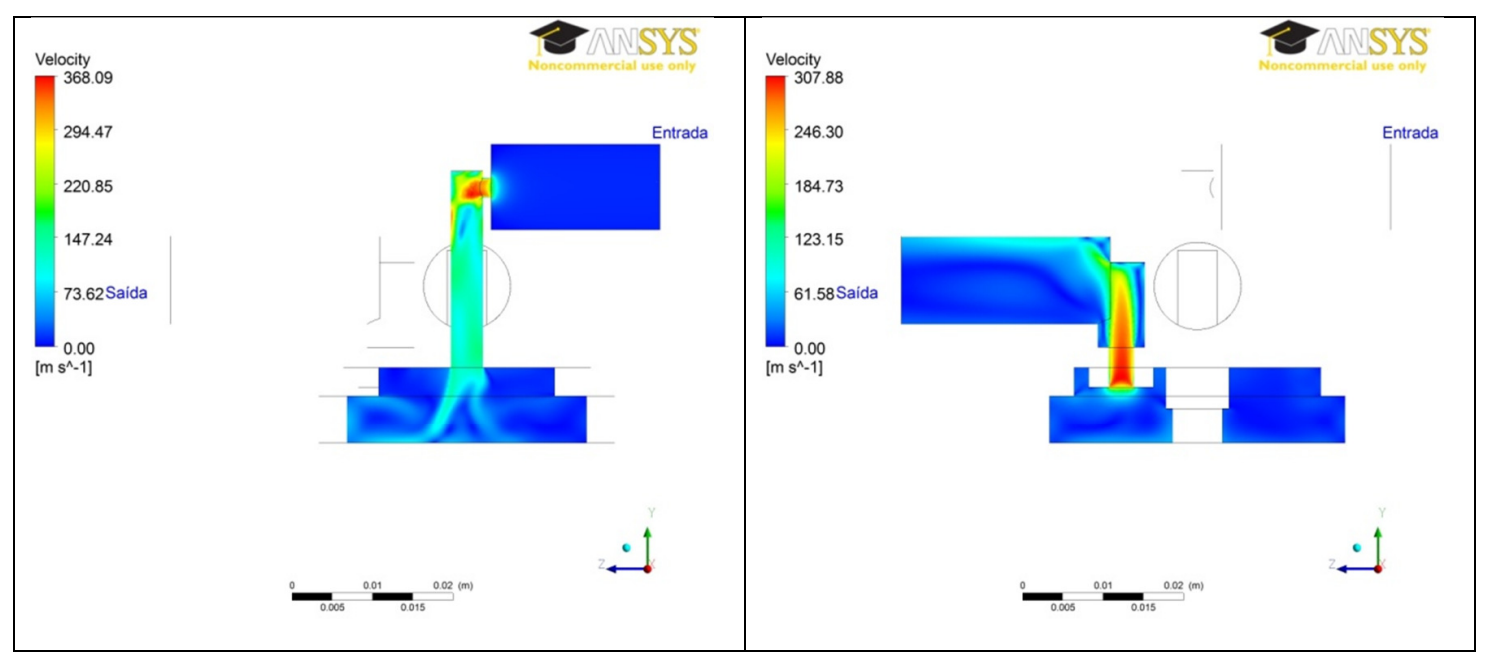

Figura 6 - Perfil de velocidade

O perfil de pressão é mostrado na Figura 7. Como esperado, as regiões com maior perda de carga são as duas regiões onde há brusca diminuição do diâmetro.

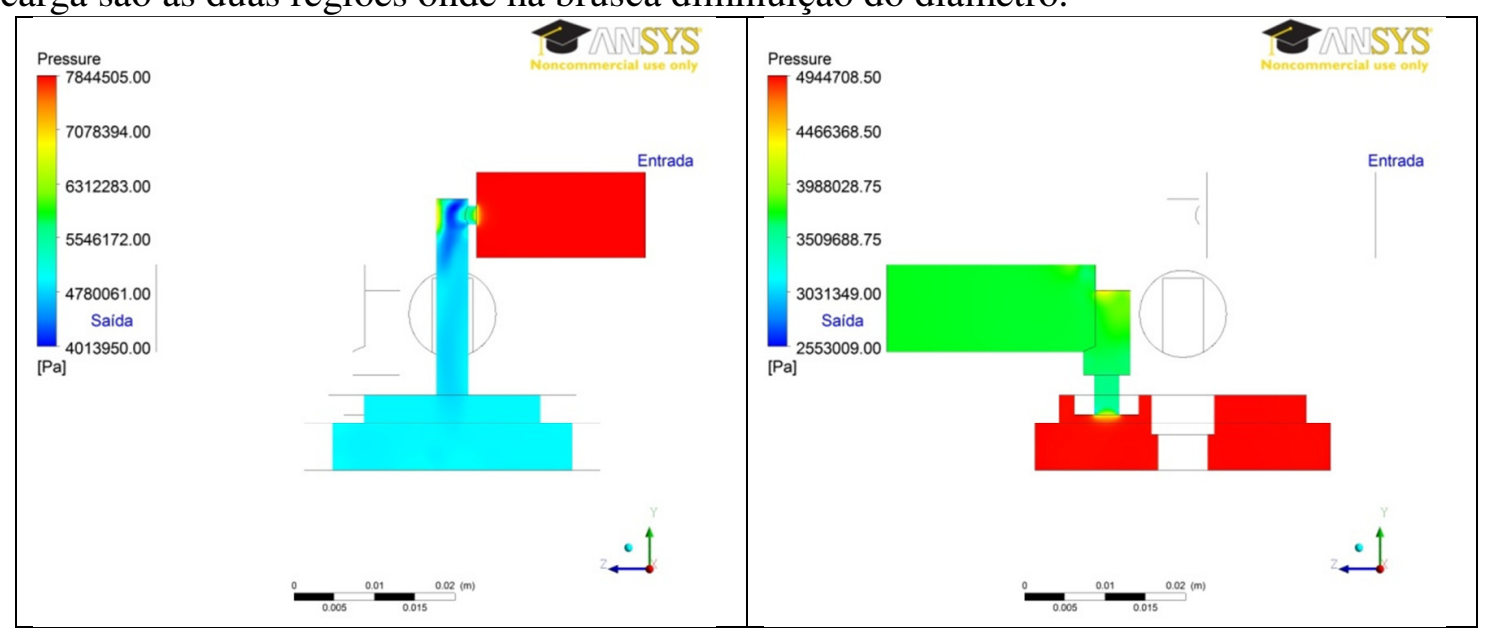

Figura 7 - Perfil de pressão 


\section{CONCLUSÕES}

O processo de deposição do enxofre em sistemas de produção e transporte de gás natural se inicia com sua supersaturação na corrente de gás. Esta supersaturação em enxofre no gás ocorre como consequência das quedas de pressão e de temperatura ocorridas, respectivamente, em função da perda de carga e da expansão volumétrica da corrente de gás devido ao efeito Joule-Thompson. Estas quedas de pressão e temperatura ocorrem com maior intensidade nas restrições como, por exemplo, em válvulas redutoras de pressão.

A partir destes resultados preliminares, pode-se afirmar que a formação e deposição do enxofre elementar nas linhas são influenciadas pela composição do gás natural e condições operacionais de pressão e de temperatura durante o transporte conforme demonstrado pelos resultados obtidos por Fluidodinâmica computacional.

\section{NOMENCLATURA}

$\Delta \mathrm{G}$ - energia livre de Gibbs (J);

$\mathrm{J}$ - taxa de nucleação $\left(\mathrm{cm}^{-3} \mathrm{~s}^{-1}\right)$;

$\mathrm{K}$ - fator pré-exponencial $\left(\mathrm{cm}^{-3} \mathrm{~s}^{-1}\right)$;

$\mathrm{k}_{\mathrm{B}}$ - constante de Boltzmann $(\mathrm{J} / \mathrm{K})$;

$\mathrm{k}_{0}$ - fator não isotérmico (=1 para misturas diluídas);

$\mathrm{M}$ - volume molar do enxofre $\left(\mathrm{mol} / \mathrm{m}^{3}\right)$;

$\mathrm{N}$ - numero de moléculas condensáveis $\left(\mathrm{cm}^{-3}\right)$;

$\mathrm{N}_{\mathrm{A}}$ - número de Avogadro (mol-1);

$\mathrm{P}_{\mathrm{c}}$ - pressão crítica $(\mathrm{MPa})$;

$\mathrm{T}$ - temperatura $(\mathrm{K})$;

$\mathrm{T}_{\mathrm{b}}$ - temperatura no ponto de bolha $(\mathrm{K})$;

$\mathrm{T}_{\mathrm{br}}$ - temperatura reduzida no ponto de bolha (K).

$\mathrm{T}_{\mathrm{c}}$ - temperatura crítica $(\mathrm{K})$;

$\mathrm{v}_{\mathrm{s}}$ - volume molecular do soluto $\left(\mathrm{m}^{3}\right)$;

$\mathrm{y}_{\mathrm{E}}$ - fração molar do soluto em condições de extração;

$\alpha_{c}$ - fator de condensação $(\mathrm{m} / \mathrm{s})$;

$\rho_{\mathrm{M}}$ - densidade da mistura $\left(\mathrm{mol} / \mathrm{cm}^{3}\right)$;

$\sigma$ - tensão interfacial do soluto $(\mathrm{N} / \mathrm{m})$;

\section{REFERÊNCIAS BIBLIOGRÁFICAS}

Lima, R.; Dias, J. S. Relatório de Análises Cromatográficas do Gás Natural do Campo de Fazenda Mamoeiro. LAPEG/UNIFACS. Salvador, 2008.

Turk, M., Influence of Thermodynamic Behaviour and Solute Properties on Homogeneous Nucleation in Supercritical Solutions. Journal of Supercritical Fluids, 18, 169-184, 2000.

Zhu, Z., Tajallipour, N.;Teevens, P. J., Modeling of Elemental Sulfur Deposition in Sour-Gas Petroleum Pipelines. Corrosion Conference and Expo., Houston, 2011. 